\title{
Pharmacokinetic drug interaction profile of omeprazole with adverse consequences and clinical risk management
}

This article was published in the following Dove Press journal:

Therapeutics and Clinical Risk Management

25 May 2013

Number of times this article has been viewed

Wei $\mathrm{Li}^{\prime}$

Su Zeng ${ }^{2}$

Lu-Shan Yu ${ }^{2}$

Quan Zhou ${ }^{3}$

'Division of Medical Affairs, Second Affiliated Hospital, School of Medicine, Zhejiang University, Hangzhou, People's Republic of China; ${ }^{2}$ Department of Pharmaceutical Analysis and Drug Metabolism, College of Pharmaceutical Sciences, Zhejiang University, Hangzhou, People's Republic of China; ${ }^{3}$ Department of Pharmacy, Second Affiliated Hospital, School of Medicine, Zhejiang University, Hangzhou, People's Republic of China

Correspondence: Quan Zhou Department of Pharmacy, Second Affiliated Hospital, School of Medicine, Jiefang Rd No. 88, Zhejiang University, Hangzhou, Zhejiang 310009 ,

People's Republic of China

Tel +86 57। 877846 I5

Fax +86 57I 87022776

Email zhouquan I42602@zju.edu.cn
Background: Omeprazole, a proton pump inhibitor (PPI), is widely used for the treatment of dyspepsia, peptic ulcer, gastroesophageal reflux disease, and functional dyspepsia. Polypharmacy is common in patients receiving omeprazole. Drug toxicity and treatment failure resulting from inappropriate combination therapy with omeprazole have been reported sporadically. Systematic review has not been available to address the pharmacokinetic drug-drug interaction (DDI) profile of omeprazole with adverse consequences, the factors determining the degree of DDI between omeprazole and comedication, and the corresponding clinical risk management.

Methods: Literature was identified by performing a PubMed search covering the period from January 1988 to March 2013. The full text of each article was critically reviewed, and data interpretation was performed.

Results: Omeprazole has actual adverse influences on the pharmacokinetics of medications such as diazepam, carbamazepine, clozapine, indinavir, nelfinavir, atazanavir, rilpivirine, methotrexate, tacrolimus, mycophenolate mofetil, clopidogrel, digoxin, itraconazole, posaconazole, and oral iron supplementation. Meanwhile, low efficacy of omeprazole treatment would be anticipated, as omeprazole elimination could be significantly induced by comedicated efavirenz and herb medicines such as St John's wort, Ginkgo biloba, and yin zhi huang. The mechanism for DDI involves induction or inhibition of cytochrome P450, inhibition of P-glycoprotein or breast cancer resistance protein-mediated drug transport, and inhibition of oral absorption by gastric acid suppression. Sometimes, DDIs of omeprazole do not exhibit a PPI class effect. Other suitable PPIs or histamine 2 antagonists may be therapeutic alternatives that can be used to avoid adverse consequences. The degree of DDIs associated with omeprazole and clinical outcomes depend on factors such as genotype status of CYP2C19 and CYP1A2, ethnicity, dose and treatment course of precipitant omeprazole, pharmaceutical formulation of object drug (eg, mycophenolate mofetil versus enteric-coated mycophenolate sodium), other concomitant medication (eg, omeprazole-indinavir versus omeprazole-indinavir-ritonavir), and administration schedule (eg, intensified dosing of mycophenolate mofetil versus standard dosing).

Conclusion: Despite the fact that omeprazole is one of the most widely prescribed drugs internationally, clinical professionals should enhance clinical risk management on adverse DDIs associated with omeprazole and ensure safe combination use of omeprazole by rationally prescribing alternatives, checking the appropriateness of physician orders before dispensing, and performing therapeutic drug monitoring.

Keywords: administration schedule, drug interactions, drug toxicity, herb-drug interactions, omeprazole, pharmacokinetics, polypharmacy, prescription auditing, risk management, treatment failure 


\section{Introduction}

Polypharmacy is a term used to describe the situation in which an individual patient is prescribed multiple medications. It often occurs because an individual patient, especially an elderly patient, may be under the care of multiple physicians: a patient who sees three different physicians may get three different prescriptions. These prescriptions may interact with each other, causing adverse drug reactions (sometimes dangerous ones) or reduced efficacy. Polypharmacy is not a problem in itself, but all too often there is a lack of coordination among care providers resulting in a risk for drug-drug interactions (DDIs). ${ }^{1-3}$

To guarantee safety in medication use, Joint Commission International requires that medication prescriptions or orders be reviewed for appropriateness before dispensing. Real or potential DDIs are one of the key elements included in the process toward appropriateness review. ${ }^{4}$ For each DDI, the object drug is defined as the medication whose pharmacokinetics and/or pharmacodynamics may be modified by the drug interaction process. The precipitant drug is defined as the medication responsible for affecting the pharmacologic action or the pharmacokinetic properties of the object drug. ${ }^{5}$

Omeprazole is a proton pump inhibitor (PPI) used in the treatment of dyspepsia, peptic ulcer disease, gastroesophageal reflux disease, laryngopharyngeal reflux, and ZollingerEllison syndrome. It is one of the most widely prescribed drugs internationally and is available over the counter in some countries. Polypharmacy is common in patients receiving omeprazole, and drug toxicity and treatment failure resulting from inappropriate combination therapy with omeprazole have been reported sporadically. DDI profiles of PPIs have been reviewed by Blume et al, Gerson and Triadafilopoulos, and Ogawa and Echizen. ${ }^{6-8}$ However, these reviews mainly address the circumstance under which DDIs occurred when PPIs were precipitant drugs, although there also were sporadic studies of DDIs that occur when omeprazole was as object drug. Meanwhile, the factors determining the degree of DDI between omeprazole and comedication, the pharmacokinetic DDI profile of omeprazole with adverse consequences, and the corresponding clinical risk management have not been addressed systematically. Therefore, we here present an updated review on this issue.

\section{Methods}

Relevant literature was identified by performing a PubMed search covering the period from January 1988 (the year omeprazole launched) to March 2013, using the search terms omeprazole and drug interaction and pharmacokinetics and additional filters (species: humans; languages: English). Three hundred and nineteen articles were detected. Inclusion criteria included studies describing omeprazole DDI with potential adverse consequences or inconsistent conclusions on clinical relevance. Exclusion criteria included studies that described omeprazole DDIs with therapeutic benefits or insignificant clinical relevance and studies that only addressed DDI issues of other PPIs, instead of omeprazole, but were still retrieved using the search terms. Sixty-three articles were finally included under this search strategy, using these inclusion/exclusion criteria.

In addition, we conducted a further review of the literature indicating that other PPIs and histamine 2 antagonists could be alternatives to omeprazole when significant DDIs occurred between omeprazole and combined medications. The full text of each article was critically reviewed, and valuable information was summarized by data interpretation.

\section{Results and discussion}

Omeprazole has actual adverse influences on pharmacokinetics of medications such as diazepam, carbamazepine, clozapine, indinavir, nelfinavir, atazanavir, rilpivirine, methotrexate (MTX), tacrolimus, mycophenolate mofetil (MMF), clopidogrel, digoxin, itraconazole, posaconazole, and oral iron supplementation. Meanwhile, low efficacy of omeprazole treatment would be anticipated, as omeprazole elimination could be significantly induced by comedicated efavirenz and herb medicines such as St John's wort, Ginkgo biloba, and yin zhi huang (YZH). The mechanism for DDI involves induction or inhibition of cytochrome P450 (CYP), inhibition of P-glycoprotein, inhibition of breast cancer resistance protein-mediated drug transport, and inhibition of oral absorption by gastric acid suppression. Sometimes, DDIs of omeprazole do not exhibit a PPI class effect. Other suitable PPIs or histamine 2 antagonists may be therapeutic alternatives to avoid adverse consequences. The degree to which DDIs are associated with omeprazole and clinical outcomes depends on many factors such as genotype status of CYP2C19 and CYP1A2, ethnicity, dose, and treatment course of precipitant omeprazole, pharmaceutical formulation of the object drug, other concomitant medication, and administration schedule. Table 1 summarizes DDIs associated with omeprazole and clinical risk management. 


\section{Circumstance I: omeprazole is comedicated as a precipitant drug Diazepam \\ DDI and risk description}

Diazepam is commonly used for treating anxiety, panic attacks, insomnia, seizures, and muscle spasms. It undergoes extensive metabolism, primarily via CYP2C19-mediated demethylation. Omeprazole $40 \mathrm{mg}$ once daily over the course of 7 days decreased diazepam plasma clearance from $22.4 \pm 2.8$ to $10.1 \pm 1.5 \mathrm{~mL} /$ hour per kilogram body weight and prolonged the elimination half-life $\left(\mathrm{t}_{1 / 2}\right)$ of diazepam from $36.9 \pm 4.1$ to $85.0 \pm 14.7$ hours. Plasma concentrations of the main metabolite desmethyldiazepam were reduced after omeprazole treatment because of CYP2C19 inhibition. ${ }^{9}$ Inhibition of CYP2C19catalyzed diazepam metabolism was estimated for omeprazole, esomeprazole, lansoprazole, dexlansoprazole, pantoprazole, and rabeprazole, using in vitro-derived inhibition parameters. The degree of inhibition varies across the PPIs, ranging from $79 \%$ (esomeprazole) and $46 \%$ (omeprazole) to less than $1 \%$ (pantoprazole and rabeprazole). ${ }^{10}$

The extent of the inhibitory effect of omeprazole on diazepam metabolism is dependent on ethnicity. The decrease in diazepam clearance and the prolongation in $\mathrm{t}_{1 / 2}$ of diazepam and desmethyldiazepam after pretreatment of omeprazole $40 \mathrm{mg}$ once daily for 21 days were significantly greater in the Caucasian group than in the Chinese group. ${ }^{11}$

\section{Clinical risk management}

Combination use of the two drugs should be avoided in case of diazepam toxicity, with adverse effects such as confusion and ataxia. Pantoprazole, lansoprazole, and rabeprazole at the recommended doses do not significantly impair the metabolism of diazepam, and thus they are alternative PPIs for patients receiving diazepam without the need to adjust diazepam dosage. ${ }^{12-14}$

Oxazepam and lorazepam are two benzodiazepines that are not oxidatively metabolized by CYP but are glucuronidated by glucuronyl transferase, and therefore they are not subject to CYP-mediated metabolic inhibition. When clinically indicated, oxazepam and lorazepam may be the benzodiazepines of choice to use together with omeprazole.

\section{Carbamazepine \\ DDI and risk description}

Carbamazepine is a widely prescribed antiepileptic drug. It undergoes extensive metabolism, primarily via
CYP3A4-mediated oxidation. It is possible for a patient with epilepsy who is receiving carbamazepine to also be receiving omeprazole because of gastrointestinal problems. Omeprazole is metabolized by CYP2C19 and CYP3A4, and carbamazepine is metabolized by CYP3A4, which can lead to competitive inhibition of carbamazepine metabolism. Multiple-dose administration of omeprazole (20 mg once daily) significantly altered the pharmacokinetics of carbamazepine in patients with duodenal ulcer (ie, increased area under the curve $\left[\mathrm{AUC}_{(0-\infty)}\right]$ from 382.3 to $668.8 \mu \mathrm{g} / \mathrm{mL}$ per hour, prolonged $\mathrm{t}_{1 / 2}$ from 17.2 to 37.3 hours, and decreased clearance from 20.7 to $12.5 \mathrm{~mL} /$ hour per kilogram body weight of carbamazepine). ${ }^{15}$ A study by Dixit et $\mathrm{al}^{16}$ noted that multiple-dose administration of omeprazole increased the peak concentrations $\left(\mathrm{C}_{\max }\right)$, $\mathrm{AUC}_{(0-\infty)}$, and $\mathrm{t}_{1 / 2}$ of sustained-release carbamazepine in healthy male volunteers. Furthermore, combination use of omeprazole and carbamazepine could lead to decreased AUC of omeprazole because of the induction of CYP3A4 by carbamazepine. ${ }^{17}$

\section{Clinical risk management}

Therapeutic drug monitoring should be carried out when carbamazepine is coadministered with omeprazole. Pantoprazole is an alternative to omeprazole, as no dose adjustment of carbamazepine is required during concomitant use of pantoprazole at $40 \mathrm{mg}$ once daily for 21 days. ${ }^{18}$ Multiple doses of ranitidine do not alter single-dose carbamazepine pharmacokinetics in healthy adults; thus, it may be an alternative choice for acid suppression. ${ }^{19}$

\section{Clozapine DDI and risk description}

Clozapine is an atypical antipsychotic drug with a narrow therapeutic range. It is metabolized primarily by CYP1A2, an important determinant of clozapine dosage in patients with schizophrenia. Omeprazole is an inducer of CYP1A2 in vivo, and its inducibility of CYP1A2 is related to the genetic polymorphism of CYP1A2 and omeprazole dose. ${ }^{20,21}$ Concomitant use of omeprazole reduced the plasma concentrations of clozapine by about $40 \%$ in patients with schizophrenia who received omeprazole in addition to clozapine because of gastrointestinal complaints. ${ }^{22}$ The changes in the pharmacokinetics of clozapine are a result of the induction of CYP1A2 by omeprazole. Mookhoek et $\mathrm{al}^{23}$ retrospectively evaluated the effect of omeprazole on clozapine metabolism in patients taking clozapine and omeprazole. 
Table I Drug-drug interactions associated with omeprazole and clinical risk management

\begin{tabular}{ll}
\hline $\begin{array}{l}\text { Drug-drug } \\
\text { interactions }\end{array}$ & $\begin{array}{l}\text { Mechanism for drug-drug } \\
\text { interactions }\end{array}$ \\
\hline $\begin{array}{l}\text { Precipitant drugs } \\
\text { Diazepam }\end{array}$ & $\begin{array}{l}\text { Omeprazole impairs CYP2CI9- } \\
\text { mediated demethylation of diazepam. }\end{array}$ \\
Carbamazepine & $\begin{array}{l}\text { Omeprazole competitively inhibits } \\
\text { CYP3A4-mediated carbamazepine } \\
\text { metabolism. } \\
\text { Omeprazole induces CYPIA2-mediated } \\
\text { clozapine metabolism. } \\
\text { Omeprazole decreases the solubility } \\
\text { Indinavir, nelfinavir, } \\
\text { atazanavir, and } \\
\text { rilpivirine }\end{array} \quad \begin{array}{l}\text { atazanavir, and rilpivirine by increasing } \\
\text { gastric pH. In addition, omeprazole } \\
\text { competitively inhibits CYP2CI9- } \\
\text { mediated formation of nelfinavir's } \\
\text { pharmacologically active metabolite. }\end{array}$
\end{tabular}

MTX

Tacrolimus

MMF

Clopidogrel

Digoxin
Omeprazole blocks the active tubular secretion of MTX by the inhibition of renal elimination of hydrogen ion, as well as MTX efflux via the breast cancerresistance protein in kidneyproximal tubules.

Omeprazole competitively inhibits a CYP3A4-mediated tacrolimus metabolism, especially in poor metabolizers for CYP2CI9.

Proton pump inhibitors reduce absorption of MMF by elevating gastric $\mathrm{pH}$ and decreasing dissolution of MMF.

Proton pump inhibitors competitively impair the metabolic activation of clopidogrel via CYP2CI9 inhibition.

Omeprazole induces the gastric permeability to digoxin and impairs the clearance by $\mathrm{P}$-glycoprotein inhibition.
Clinical risk management

I. Avoid such combination use in case of diazepam toxicity.

2. Pantoprazole and lansoprazole are alternative proton pump inhibitors. Oxazepam and lorazepam are alternatives to use together with omeprazole.

I. Therapeutic drug monitoring should be carried out when carbamazepine is coadministered with omeprazole.

2. Select pantoprazole as an alternative proton pump inhibitor.

I. Close monitoring of plasma clozapine levels is recommended.

2. Select pantoprazole as an alternative proton pump inhibitor.

I. Indinavir: concomitant $\mathbf{2 0 0} \mathrm{mg}$ ritonavir therapy may overcome the significant omeprazole-indinavir DDI. Cimetidine is an alternative to omeprazole.

2. Nelfinavir: combination use of nelfinavir and proton pump inhibitor may be acceptable for indications where the proton pump inhibitor is required for fewer than 30 days.

3. Atazanavir: increasing the atazanavir/ritonavir dose to $400 / 100 \mathrm{mg}$ can attenuate the effect of omeprazole and warrant enough antiviral effect against wild-type HIV. Another option is to select alternative strategies for anti-HIV treatment that have a minimal risk of DDI with omeprazole.

4. Rilpivirine: famotidine may be an alternative to omeprazole if spaced appropriately (ie, famotidine is administered 12 hours before or 4 hours after rilpivirine).

I. Close therapeutic drug monitoring should be performed for patients receiving high-dose MTX therapy so as to decide whether to initiate the calcium folinate rescue therapy.

2. A histamine 2 antagonist is recommended to substitute for a proton pump inhibitor, as concurrent therapy does not result in MTX toxicity.

I. Close therapeutic drug monitoring of tacrolimus should be carried out when starting or switching a proton pump inhibitor.

2. Rabeprazole and pantoprazole are alternatives to omeprazole.

I. Pay more attention to monitoring mycophenolic acid levels in the presence of omeprazole, especially in the first week posttransplantation.

2. Adopt a new strategy; that is, intensified dosing of MMF ( $1.5 \mathrm{~g}$ twice daily on days $\mathrm{I}-5$, then $\mathrm{I} .0 \mathrm{~g}$ twice daily) instead of standard dosing ( $1.0 \mathrm{~g}$ twice daily).

3. Enteric-coated mycophenolate sodium may be an alternative to MMF.

I. Pantoprazole and rabeprazole are alternatives to omeprazole.

2. PA32540 (a single-tablet formulation of $325 \mathrm{mg}$ aspirin and $40 \mathrm{mg}$ immediate-release omeprazole) and clopidogrel spaced 10 hours apart is a good strategy in comparison with synchronous administration of aspirin, clopidogrel, and omeprazole.

I. Pantoprazole is an alternative to omeprazole when combination use of proton pump inhibitor and digoxin is needed.

2. Monitor the serum concentrations and toxicity symptoms of digoxin in the presence of omeprazole, and adjust the dose of digoxin as needed. 
Table I(Continued)

\begin{tabular}{|c|c|c|}
\hline $\begin{array}{l}\text { Drug-drug } \\
\text { interactions }\end{array}$ & $\begin{array}{l}\text { Mechanism for drug-drug } \\
\text { interactions }\end{array}$ & Clinical risk management \\
\hline $\begin{array}{l}\text { Itraconazole, } \\
\text { posaconazole }\end{array}$ & $\begin{array}{l}\text { Omeprazole reduces the } \mathrm{pH} \text {-dependent } \\
\text { absorption of itraconazole and } \\
\text { posaconazole by suppressing gastric } \\
\text { secretion. }\end{array}$ & $\begin{array}{l}\text { I. Avoid using proton pump inhibitors for patients receiving } \\
\text { itraconazole or posaconazole. } \\
\text { 2. Monitor serum levels of posaconazole or switch to an } \\
\text { alternative antifungal therapy if combination with omeprazole } \\
\text { cannot be avoided. Histamine } 2 \text { antagonists have less } \\
\text { interaction with posaconazole than proton pump inhibitors. } \\
\text { 3. Coadministration of an acidic solution (eg, a cola beverage) can } \\
\text { counteract the adverse effect of gastric acid suppressants on } \\
\text { the bioavailability of itraconazole. }\end{array}$ \\
\hline $\begin{array}{l}\text { Oral iron } \\
\text { supplementation }\end{array}$ & $\begin{array}{l}\text { Omeprazole decreases the absorption } \\
\text { of oral iron supplementation. }\end{array}$ & $\begin{array}{l}\text { Iron-deficient patients taking proton pump inhibitors may have to } \\
\text { be treated with high-dose iron therapy for a longer duration or } \\
\text { with intravenous iron therapy. }\end{array}$ \\
\hline \multicolumn{3}{|l|}{ Object drug } \\
\hline $\begin{array}{l}\text { Efavirenz, herbal } \\
\text { medicines (St John's } \\
\text { wort, Ginkgo biloba, } \\
\text { yin zhi huang) }\end{array}$ & $\begin{array}{l}\text { Efavirenz, St John's wort, and Yin zhi } \\
\text { huang induce CYP2CI9 and CYP3A } \\
\text { activity and enormously accelerate } \\
\text { omeprazole elimination. Ginkgo biloba } \\
\text { can induce CYP2CI9-mediated } \\
\text { hydroxylation of omeprazole. }\end{array}$ & $\begin{array}{l}\text { It seems inappropriate to prescribe omeprazole for patients } \\
\text { receiving efavirenz, St John's wort, Ginkgo biloba, or yin zhi } \\
\text { huang. Rabeprazole is an alternative proton pump inhibitor to } \\
\text { omeprazole. }\end{array}$ \\
\hline
\end{tabular}

Abbreviations: CYP, cytochrome P450; DDI, drug-drug interaction; HIV, human immunodeficiency syndrome; MTX, methotrexate; MMF, mycophenolate mofetil.

After discontinuation of omeprazole therapy, all nonsmoking patients experienced a significant increase in serum clozapine concentrations compared with smokers, despite an unchanged daily dose of clozapine. The underlying mechanism for this interesting change was the disappearance of enzyme induction of CYP1A2 by omeprazole in nonsmokers after stopping omeprazole therapy, whereas CYP1A2 remained induced by smoking in patients who smoked.

\section{Clinical risk management}

If patients are receiving omeprazole as comedication, close monitoring of plasma clozapine levels is recommended. Pantoprazole lacks induction of CYP1A2 activity in man, ${ }^{24}$ and it may be an alternative to omeprazole when a PPI is added to clozapine therapy. However, caution should be exercised when omeprazole is replaced by pantoprazole and smoking cessation is required during hospitalization of smoking patients with psychosis who have been receiving omeprazole and clozapine. Otherwise, a strong increase in plasma clozapine concentrations will be observed because of the loss of the inducible effects on CYP1A2 of both smoking and omeprazole..$^{25}$

\section{Anti-HIV-I agents}

The use of acid suppressants among human immunodeficiency virus (HIV)-infected patients is common and may potentially generate clinically significant DDIs that alter plasma concentrations of anti HIV-1 agents. It is important to understand the risk of these interactions, thereby maximizing antiviral potential and preventing HIV antiretroviral resistance. ${ }^{26}$

\section{DDI and risk description}

As indicated by a retrospective analysis, patients receiving a combination of indinavir ( $800 \mathrm{mg}$ three times daily) and omeprazole (20-40 mg daily) were susceptible to a lower plasma indinavir AUC than the average expected value in patients receiving indinavir alone. ${ }^{27}$ Such pharmacokinetic changes can be explained by decreased indinavir solubility and absorption resulting from increasing gastric $\mathrm{pH}$ introduced by omeprazole.

Nelfinavir is metabolized mainly via CYP2C19 to nelfinavir hydroxy-t-butylamide, which exhibits potent antiviral activity. Omeprazole coadministration (40 mg daily for 4 days) significantly decreases the AUC values of nelfinavir in healthy participants. The main mechanism for this interaction was the suppression of gastric acid secretion, resulting in reduced nelfinavir solubility, and competitive inhibition of CYP2C19 by omeprazole, resulting in a reduction in pharmacologically active nelfinavir hydroxy-t-butylamide. Hence, omeprazole should not be coadministered to patients taking nelfinavir. ${ }^{28}$

Concomitant use of omeprazole and atazanavir is currently not recommended. Omeprazole $40 \mathrm{mg}$ once daily significantly decreased atazanavir exposure by approximately $75 \%$. 
Even if atazanavir/ritonavir 300/100 mg were used, the addition of omeprazole still reduced atazanavir AUC and trough concentrations by $42 \%$ and $46 \%$, respectively. ${ }^{29}$ Rilpivirine, a non-nucleoside reverse transcriptase inhibitor, may have pharmacokinetic interactions with inducers and inhibitors of CYP3A4, which are drugs that increase gastric $\mathrm{pH}$, and P-glycoprotein substrates. Rilpivirine should not be coadministered with PPIs in case of a significant reduction in rilpivirine absorption. ${ }^{30}$

\section{Clinical risk management}

With respect to significant omeprazole-indinavir DDIs, concomitant $200 \mathrm{mg}$ ritonavir therapy may reverse this adverse interaction, as it can substantially inhibit clearance of indinavir, presumably via mechanism-based inhibition of CYP3A-mediated metabolism of indinavir or, possibly, inhibition of P-glycoprotein. ${ }^{31-33}$ As described in the package insert for Crixivan ${ }^{\circledR}$ (indinavir sulfate; Merck and Co, Inc, Whitehouse Station, NJ, USA), comedicated histamine 2 antagonist cimetidine ( $600 \mathrm{mg}$ twice daily) minimally affected the AUC of a single $400 \mathrm{mg}$ dose of indinavir. In situations in which both indinavir and cimetidine are indicated, cimetidine is an alternative in that its potent inhibitory effect on CYP3A may offset the influence of the elevated $\mathrm{pH}$ changes. ${ }^{34}$

For risk management of nelfinavir-omeprazole DDIs, it was suggested that combination use of nelfinavir and a PPI may be acceptable for indications in which the PPI is required for fewer than 30 days. A retrospective cohort study in 1147 HIV-positive adults receiving nelfinavir showed that the risk for virologic rebound was closely related with the time of exposure to the PPI. The hazard of virologic rebound resulting from short-term use of PPIs (defined as within 30 days of initial PPI dispensation) was 1.07 compared with no PPI exposure, whereas there was a $56 \%$ increased risk with longterm ( $\geq 30$ days) exposure to PPIs compared with the risk in the unexposed group $(P=0.02){ }^{35}$

As far as risk management on atazanavir-omeprazole DDIs is concerned, increasing the atazanavir/ritonavir dose to $400 / 100 \mathrm{mg}$ can attenuate the effect of omeprazole and warrant enough antiviral effect against wild-type $\mathrm{HIV}^{29}$ Another option is to select alternative strategies for anti-HIV treatment that have minimal risk for DDIs with omeprazole. For example, amprenavir plasma exposures were not altered when fosamprenavir (prodrug of amprenavir) and ritonavir $1400 / 200 \mathrm{mg}$ were administered once a day in the morning and $20 \mathrm{mg}$ omeprazole was given in the evening. ${ }^{36}$

If spaced appropriately, famotidine may be an alternative to PPIs for patients receiving rilpivirine. When famotidine
$40 \mathrm{mg}$ was administered 12 hours before or 4 hours after rilpivirine, rilpivirine pharmacokinetics were not significantly affected; such combination use is acceptable..$^{37,38}$

\section{Methotrexate}

\section{DDI and risk description}

MTX is an antifolate agent used in the treatment of various cancers and some autoimmune diseases. It is frequently administered at a high dose in oncology and comes with various procedures to reduce the occurrence of toxicity and, particularly, to ensure optimal renal elimination. MTX is actively secreted in the distal tubules. A probable drug interaction between omeprazole and MTX was observed according to the Naranjo probability scale. ${ }^{39}$ The proposed mechanism is that omeprazole can block the active tubular secretion of MTX through inhibition of renal elimination of the hydrogen ion and can inhibit breast cancer resistance protein-mediated efflux of MTX in human kidney proximal tubules. PPI coadministration independently increased the risk of delayed MTX elimination by 2.65 times. $^{40}$

\section{Clinical risk management}

Close therapeutic drug monitoring should be performed for patients receiving high-dose MTX therapy so as to decide whether to initiate the calcium folinate rescue therapy. A histamine 2 antagonist is recommended to substitute for a PPI, as concurrent therapy does not result in MTX toxicity. ${ }^{41}$ For patients receiving high-dose MTX, transient discontinuation of the PPI or a switch for ranitidine should be proposed to avoid severe DDI, and similarly, a warning should be implemented when ranitidine $(150 \mathrm{mg})$ is switched to omeprazole (20 mg daily, orally). ${ }^{42}$

\section{Tacrolimus}

\section{DDI and risk description}

Tacrolimus, an immunosuppressive drug, undergoes extensive hepatic metabolism largely via CYP3A4. In vitro studies using human liver microsomes have shown that omeprazole inhibits CYP3A4-mediated metabolism of tacrolimus competitively. ${ }^{43}$ In the case of poor metabolizers (PMs) for CYP2C19, or if high doses of omeprazole (40 mg) are given to extensive metabolizers (EMs), CYP3A4 becomes the main enzyme for omeprazole elimination. The shared metabolism of omeprazole and tacrolimus through CYP3A4 has been associated with clinically significant drug interactions, especially in patients who are classified as PMs for CYP2C19. ${ }^{44}$ The CYP2C19 polymorphisms, both in the native intestine and in the graft liver, have an effect on the 
interaction between tacrolimus and omeprazole in adult living-donor liver transplant patients. The concentration/ dose ratio of tacrolimus coadministered with omeprazole was significantly higher in patients with two variant alleles for CYP2C19 than those with the wild-type homozygote $($ CYP2C $19 * 1 / * 1)$ or heterozygote $\left(\mathrm{CYP} 2 \mathrm{C} 19^{*} 1 / * 2\right.$ or CYP2C19*1/*3) $(P=0.010$ for native intestine; $P=0.022$ for graft liver). ${ }^{45}$

\section{Clinical risk management}

Close therapeutic drug monitoring of tacrolimus should be considered when starting or switching a PPI in organ transplant recipients receiving tacrolimus-based immunosuppression. Esomeprazole and lansoprazole are also susceptible to interactions with tacrolimus. ${ }^{44,46}$ Concomitant administration of rabeprazole or pantoprazole has insignificant influence on the pharmacokinetics of tacrolimus in adult transplant patients. ${ }^{47-49}$

\section{Mycophenolate mofetil DDI and risk description}

MMF, an immunosuppressant and prodrug of mycophenolic acid (MPA), is used extensively in transplant medicine. MPA exposure by AUC correlates with the incidence of acute rejection episodes and transplant vasculopathy. Combination use of PPIs and MMF is possible because gastrointestinal adverse effects are common after organ transplantation. Unfortunately, PPI comedication could reduce active drug exposure in heart transplant recipients and renal transplant patients, thereby increasing the risk for treatment failure. ${ }^{50,51}$

\section{Clinical risk management}

Optimization of administration schedule may be an option for managing DDIs between MMF and PPIs. Kiberd et $\mathrm{al}^{52}$ presented a new finding that MPA pharmacokinetics were not significantly affected when an intensified dosing of MMF (1.5 g twice daily on days $1-5$, then $1.0 \mathrm{~g}$ twice daily) instead of standard dosing ( $1.0 \mathrm{~g}$ twice daily) was used in combination with PPI therapy. This strategy may warrant adequate MPA exposure, whether or not a patient receives PPI comedication.

Omeprazole impairs the absorption of MMF, but not enteric-coated mycophenolate sodium (a formulation therapeutically equivalent to MMF), in healthy volunteers, indicating that impaired absorption of MMF with concomitant PPI is a result of incomplete dissolution of MMF in the stomach at elevated $\mathrm{pH} .{ }^{53}$ Therefore, enteric-coated mycophenolate sodium may be an alternative to immunosuppressant treatment.
David-Neto et $\mathrm{a}^{51}$ presented the valuable finding that the mean $\mathrm{AUC}_{(0-12 \mathrm{~h})}$ of MPA was in the lower limit of the therapeutic window on day 7 in patients using omeprazole but quickly increased on day 14 and thereafter, and that most patients were within the therapeutic window after day 7. This information is important for clinicians because there is a decreased incidence of acute rejection when the patients are adequately exposed to MPA during the first week. Clinicians should pay more attention to monitoring MPA levels in the presence of omeprazole, especially in the first week post-transplantation.

\section{Clopidogrel}

\section{DDI and risk description}

Clopidogrel is an oral antiplatelet agent used to inhibit blood clots in coronary artery disease, peripheral vascular disease, and cerebrovascular disease. Clopidogrel, along with aspirin, is considered the gold standard treatment for acute coronary syndrome by reducing the risk for new ischemic events; however, the dual therapy increases the risk for gastrointestinal bleeding. Therefore, comedicated PPI is recommended by most guidelines. Clopidogrel is a prodrug that needs to be converted to an active metabolite via hepatic activation, mainly through CYP2C19 and CYP3A4. As PPI metabolism also involves CYP2C19, it was assumed that PPIs might competitively interfere with clopidogrel's action. ${ }^{54,55}$

Most of the literature suggests that omeprazole is the PPI most likely to have a significant interaction with clopidogrel. However, in the Clopidogrel with or without Omeprazole in Coronary Artery Disease (COGENT) study, the authors randomly assigned patients $(\mathrm{n}=3873)$ with an indication for dual antiplatelet therapy to receive clopidogrel in combination with either omeprazole or placebo in addition to aspirin. It was concluded that prophylactic use of omeprazole reduced the rate of upper gastrointestinal bleeding (hazard ratio with omeprazole, $0.13 ; P=0.001)$ and that there was no apparent cardiovascular interaction between the two drugs (hazard ratio with omeprazole, $0.99 ; P=0.96) .{ }^{56}$

\section{Clinical risk management}

To reduce the potential DDI between PPIs and clopidogrel, one option is to select a PPI that demonstrates relatively less influence on CYP2C19. Indeed, the clopidogrel-PPI interaction does not exhibit a PPI class effect. ${ }^{57}$ Coadministration of rabeprazole and clopidogrel did not affect the antiplatelet efficacy of clopidogrel in healthy Chinese volunteers. ${ }^{58}$ Neubauer et a $l^{59}$ concluded that pantoprazole did not diminish the antiplatelet efficacy of clopidogrel. In situations in 
which both clopidogrel and a PPI are indicated, pantoprazole should be used, as it is the PPI least likely to interact with clopidogrel.

Recently, a randomized study indicated that the use of dexlansoprazole or lansoprazole, rather than esomeprazole or omeprazole, could minimize the potential of PPIs to attenuate the efficacy of clopidogrel.$^{60}$ Concomitant use of famotidine $40 \mathrm{mg}$ daily did not reduce the platelet inhibitory effect of clopidogrel in patients under dual antiplatelet therapy; ${ }^{61}$ however, famotidine has not been proven effective for the prevention of upper gastrointestinal complications in patients receiving dual antiplatelet therapy. ${ }^{62}$ Therefore, further investigation into the feasibility of switch to famotidine is needed.

Very recently, spaced administration of a clopidogrel and a single-tablet formulation of $325 \mathrm{mg}$ aspirin and $40 \mathrm{mg}$ immediate-release omeprazole (PA32540) was considered as an alternative that might reduce the potential pharmacodynamic interaction between omeprazole and clopidogrel. The spaced administration strategy involves a morning dosing of a single tablet of PA32540 followed 10 hours later by a 300-mg clopidogrel load on day 1 , and then a morning dosing of a single tablet of PA32540 followed 10 hours later by $75 \mathrm{mg}$ clopidogrel on day 2 and thereafter. The synchronous administration strategy is that $300 \mathrm{mg}$ clopidogrel, $81 \mathrm{mg}$ aspirin, and $40 \mathrm{mg}$ omeprazole are dosed synchronously on day 1 , and then $75 \mathrm{mg}$ clopidogrel, $81 \mathrm{mg}$ aspirin, and $40 \mathrm{mg}$ omeprazole are given synchronously on day 2 and thereafter. The spaced therapy had greater antiplatelet effects than did the synchronous administration strategy $(P=0.004){ }^{63}$

\section{Digoxin}

\section{DDI and risk description}

Digoxin is a medication widely used in treating heart failure. DDIs with digoxin are important because of this agent's narrow therapeutic index. The usual digoxin therapeutic range is $0.8-2 \mathrm{ng} / \mathrm{mL}$. Omeprazole-associated digoxin toxicity has been observed in a 65 -year-old Caucasian woman. ${ }^{64}$ The patient was treated with digoxin at a dose of $0.625 \mathrm{mg}$ daily for 6 years. Three months after initiation of omeprazole therapy (20 mg once daily), her serum digoxin level jumped from 1.1 to $3.9 \mathrm{ng} / \mathrm{mL}$ with normal hepatic and renal function. The possible mechanism for such an interaction may involve increased stomach absorption and impaired clearance by inhibition of P-glycoprotein. Omeprazole can induce the gastric permeability to digoxin, ${ }^{65}$ and it can dosedependently increase absorptive (apical-to-basal) digoxin permeability with maximal increases of 2.6-fold across human colonic carcinoma cell line (Caco-2) ${ }^{66}$ Omeprazole inhibits P-glycoprotein-mediated digoxin transport in Caco-2 cells with a half maximal inhibitory concentration $\left(\mathrm{IC}_{50}\right)$ value of $17.7 \mu \mathrm{M}$. In EMs of CYP2C19, the low dose of $20 \mathrm{mg}$ omeprazole will result in a $\mathrm{C}_{\max }$ of $1.5-3.5 \mu \mathrm{M}$. In PMs of CYP2C19, plasma concentrations of omeprazole, especially at the $40 \mathrm{mg}$ dose, would even reach the observed $\mathrm{IC}_{50}$ value. Therefore, it is assumed that PMs of CYP2C19 will be especially susceptible to the omeprazole/digoxin interaction. ${ }^{67}$

\section{Clinical risk management}

Patients should be monitored for digoxin serum concentrations and signs of digoxin toxicity in the presence of omeprazole, and doses should be adjusted as needed. Pantoprazole $40 \mathrm{mg}$ once daily for 5 days does not exhibit significant DDIs with digoxin in healthy volunteers, and these drugs may be administered concomitantly without the need for dose adjustment. ${ }^{68}$

\section{Antifungal agents \\ DDI and risk description}

The absorption of itraconazole is dependent on gastric acidity. Concomitant omeprazole treatment (40 mg once daily) for 2 weeks can significantly reduce the $\mathrm{AUC}_{0-24}$ and $\mathrm{C}_{\max }$ of oral itraconazole capsules by $64 \%$ and $66 \%$, respectively. ${ }^{69}$ However, omeprazole $40 \mathrm{mg}$ once daily for 7 days has no significant effect on the $\mathrm{C}_{\text {max }}$ of itraconazole oral solution. ${ }^{70}$ This pharmacokinetic DDI difference may be attributed to the fact that itraconazole oral solution improved bioavailability and reduced $\mathrm{pH}$ dependency for adequate absorption when compared with capsules.

Posaconazole is an extended-spectrum triazole antifungal agent for the prevention and treatment of invasive fungal infections. Elevated gastric $\mathrm{pH}$ is accompanied by decreased posaconazole absorption. Omeprazole (40 mg once daily) for 3 days could significantly reduce posaconazole serum trough level. ${ }^{71}$ When a higher dosage of omeprazole is used, this adverse effect could be profound and may result in therapeutic failure.

\section{Clinical risk management}

Omeprazole and itraconazole capsule should not be used together. The two itraconazole formulations contain the same active ingredient, and it seems encouraging to substitute itraconazole oral solution for itraconazole capsule when coadministered with omeprazole. However, as described in package inserts, the two products have different indications and should not be used interchangeably. Fortunately, the 
adverse effect of gastric acid suppressants on the bioavailability of itraconazole can be counteracted by the coadministration of an acidic solution (eg, a cola beverage) that transiently reduces the gastric $\mathrm{pH}^{72}$

With respect to risk management of omeprazole-posaconazole DDIs, either serum levels of posaconazole should be monitored or antifungal therapy should be switched to an alternative agent if comedicated omeprazole cannot be avoided. ${ }^{71}$ In patients with subtherapeutic posaconazole concentrations, increased dose frequency, administration with high-fat meals, and cessation of interacting medication use are useful strategies to improve systemic absorption. ${ }^{73}$ Histamine 2 antagonists may have less interaction with posaconazole than PPIs. Posaconazole target attainment was associated with the use of histamine 2 antagonists over PPIs (odds ratio, 6.8). ${ }^{74}$

\section{Oral iron supplementation}

Omeprazole, and possibly all PPIs, can decrease the absorption of oral iron supplementation. Physicians should be cognizant of an inadequate response to oral iron supplements by patients receiving PPIs. Iron-deficient patients taking PPIs may have to be treated either with high-dose iron therapy for a longer duration or with intravenous iron therapy. ${ }^{75}$

\section{Circumstance 2: omeprazole is comedicated as an object drug}

Omeprazole is extensively metabolized in the liver through CYP2C19-medicated 5-hydroxylation and CYP3A4medicated sulfoxidation reactions. ${ }^{76}$ The low efficacy of the omeprazole treatment will be anticipated, as omeprazole elimination could be significantly induced by the following comedications.

\section{Efavirenz}

Efavirenz is a non-nucleoside reverse transcriptase inhibitor of HIV-1 reverse transcriptase used for the treatment of acquired immunodeficiency syndrome. In vitro studies have demonstrated that efavirenz could activate CYP3A4 and CYP2C19 promoter activity through the pregnane X receptor and the constitutive androstane receptor. ${ }^{77-79}$ Repeated administration of efavirenz $600 \mathrm{mg}$ daily can induce CYP2C19 and CYP3A activity and significantly decrease the AUC ${ }_{(0-\infty)}$ of omeprazole (about twofold) in healthy volunteers. ${ }^{80}$

\section{St John's wort}

St John's wort, an extract of the medicinal plant Hypericum perforatum, is widely used as a herbal antidepressant.
Receiving a $300 \mathrm{mg}$ St John's wort tablet three times daily for 14 days can induce both CYP3A4-catalyzed sulfoxidation and CYP2C19-dependent hydroxylation of omeprazole, which enormously decreases the plasma concentrations of omeprazole. Omeprazole's bioavailability was significantly reduced in CYP2C19-genotyped EMs compared with PMs after long-term use of St John's wort. ${ }^{81,82}$

\section{Ginkgo biloba}

Ginkgo biloba is one of the most popular herbal medicines in the world. It has demonstrated remarkable pharmacological actions such as memory enhancing, cognition improving, and antiplatelet effects. Pharmacokinetic studies in healthy volunteers presented no herb-drug interaction between Ginkgo biloba extract and CYP2C19 substrates such as diazepam and voriconazole, indicating no evidence of induction of CYP2C19 activity by Ginkgo biloba treatment. ${ }^{83,84}$ A cocktail interaction study in healthy volunteers demonstrated no relevant effect of Ginkgo biloba extract (240 mg daily for 8 days) on the in vivo activity of the major CYP enzymes in humans. ${ }^{85}$ The first report of induction of CYP2C19mediated hydroxylation by Ginkgo biloba in humans was presented by Yin et al. ${ }^{86}$ Ginkgo biloba treatment $(140 \mathrm{mg}$ twice daily) for 12 days induced omeprazole hydroxylation in a CYP2C19 genotype-dependent manner (ie, the inducible effect was greater in PMs than EMs). The $\mathrm{AUC}_{(0-\infty)}$ ratios of omeprazole to 5-hydroxyomeprazole (an index of CYP2C19 activity) were decreased $42.3 \%, 50.3 \%$, and $70.6 \%$ $(P<0.01)$ in the homozygous EMs, heterozygous EMs, and PMs, respectively. Plasma concentrations of omeprazole and omeprazole sulfone were significantly decreased, and concentrations of 5-hydroxyomeprazole were significantly increased, after Ginkgo biloba administration. The observed interaction between Ginkgo biloba and omeprazole may be clinically important. The clinical efficacy and reduction of acid secretion with omeprazole is related to individual exposure to omeprazole.

\section{$\mathrm{YZH}$}

Herbal medicine YZH, a decoction of yin chin (Artemisia capillaris) and three other herbs, is widely used in Asia to prevent and treat jaundice. Huang et $\mathrm{al}^{87}$ demonstrated that $\mathrm{YZH}$ activated the constitutive androstane receptor (constitutive androstane receptor, NR1I3), which further increases CYP3A4 and CYP2C19 expression. In clinical use, YZH and omeprazole are often coadministrated by patients with liver cirrhosis. Treatment of YZH oral liquid (10 $\mathrm{mL}$ three times daily for 14 days) enormously decreased 
plasma concentrations of omeprazole and greatly increased those of omeprazole sulfone and 5-hydroxyomeprazole, indicating that YZH could induce CYP3A4-mediated sulfoxidation and CYP2C19-dependent hydroxylation of omeprazole. ${ }^{88} \mathrm{CYP} 2 \mathrm{C} 19 * 2$ homozygotes display a large degree of induction by YZH compared with CYP2C19*1/*1, CYP2C19*1/*2, and CYP2C19*1/*3 participants. AUC ${ }_{(0-\infty)}$ of omeprazole decreased by $46.69 \% \pm 17.82 \%(P=0.007)$, $41.38 \% \pm 14.04 \%(P=0.002)$, and $16.25 \% \pm 12.18 \%$ $(P=0.039)$ in CYP2C19*1/CYP2C19*1, CYP2C19*1/ CYP2C19*2 or *3, and CYP2C19*2/CYP2C19*2 participants, respectively. It can be postulated that $\mathrm{YZH}$ may lead to therapeutic failure or insufficient curative effect toward omeprazole in clinical situations because of pharmacokinetic variations.

\section{Clinical risk management}

It seems inappropriate to prescribe omeprazole for patients receiving efavirenz, St John's wort, Ginkgo biloba, or YZH. Conversely, rabeprazole is metabolized mainly through a nonenzymatic pathway to rabeprazole-thioether and, to a much lesser extent, by CYP2C19 (demethylated rabeprazole) and CYP3A4 (rabeprazole-sulfone). Therefore, rabeprazole has a lower potential for DDIs. ${ }^{89,90}$ It is assumed that DDIs between rabeprazole and any of the precipitant drugs mentioned earlier are absent, but this hypothesis needs to be verified by further investigation.

\section{Conclusion}

In this review, we specially addressed DDIs associated with omeprazole with adverse consequences, the mechanisms for DDIs, the factors determining degree of DDI, and clinical risk management. Despite the fact that omeprazole is one of the most widely prescribed drugs internationally, clinical professionals should enhance clinical risk management on adverse DDIs associated with omeprazole and ensure safe combination use of omeprazole by rationally prescribing alternatives, checking the appropriateness of physician orders before dispensing, and performing therapeutic drug monitoring.

Suggestions for future research include that the quality of DDI studies be improved. For example, according to the Preferred Reporting Items for Systematic Reviews and Meta-Analyses (PRISMA) guidelines, studies can be classified into high-quality (well-performed randomized clinical trials), moderate-quality (post hoc analysis of randomized clinical trials and propensity-matched studies), and low-quality (observational studies without propensity matching) studies. ${ }^{91}$ Meta-analyses report an inverse correlation between clopidogrel-PPI interaction and study quality $(P=0.007)$, with high-quality and moderate-quality studies not reporting any association, increasing concerns about unmeasured confounders biasing the low-quality studies. ${ }^{54}$ Adequately powered randomized controlled trials with pharmacodynamic evaluation are still needed to confirm the persisting doubts about the DDIs associated with omeprazole. In addition, investigations into factors determining the degree of DDIs as well as outcomes of clinical risk management and clinical pharmacy interventions should be strengthened.

\section{Acknowledgments}

This work was supported by Zhejiang Provincial Bureau of Health grant 2012KYA090, National Natural Science Foundation of China grant 30873122, and National Major Projects of China grants 2012ZX09506001-004 and 2009ZX09304-003.

\section{Disclosure}

The authors report no conflicts of interest in this work.

\section{References}

1. Corsonello A, Pedone C, Corica F, Incalzi RA. Polypharmacy in elderly patients at discharge from the acute care hospital. Ther Clin Risk Manag. 2007;3(1):197-203.

2. Koh Y, Kutty FB, Li SC. Drug-related problems in hospitalized patients on polypharmacy: the influence of age and gender. Ther Clin Risk Manag. 2005;1(1):39-48.

3. Doan J, Zakrzewski-Jakubiak H, Roy J, Turgeon J, Tannenbaum C. Prevalence and risk of potential cytochrome P450-mediated drug-drug interactions in older hospitalized patients with polypharmacy. Ann Pharmacother. 2013;47(3):324-332.

4. Joint Commission International. JCI accreditation standards for hospitals [webpage on the Internet]. Oak Brook, IL: Joint Commission International; 2010. Available from: http://www.jointcommissioninternational.org/common/pdfs/jcia/IAS400_Standards_Lists_Only.pdf. Accessed March 20, 2013.

5. Mahmood M, Malone DC, Skrepnek GH, et al. Potential drug-drug interactions within Veterans Affairs medical centers. Am J Health Syst Pharm. 2007;64(14):1500-1505.

6. Ogawa R, Echizen H. Drug-drug interaction profiles of proton pump inhibitors. Clin Pharmacokinet. 2010;49(8):509-533.

7. Blume H, Donath F, Warnke A, Schug BS. Pharmacokinetic drug interaction profiles of proton pump inhibitors. Drug Saf. 2006;29(9):769-784.

8. Gerson LB, Triadafilopoulos G. Proton pump inhibitors and their drug interactions: an evidence-based approach. Eur J Gastroenterol Hepatol. 2001;13(5):611-616.

9. Gugler R, Jensen JC. Omeprazole inhibits oxidative drug metabolism. Studies with diazepam and phenytoin in vivo and 7-ethoxycoumarin in vitro. Gastroenterology. 1985;89(6):1235-1241.

10. Zvyaga T, Chang SY, Chen C, et al. Evaluation of six proton pump inhibitors as inhibitors of various human cytochromes P450: focus on cytochrome P4502C19. Drug Metab Dispos. 2012;40(9):1698-1711.

11. Caraco Y, Tateishi T, Wood AJ. Interethnic difference in omeprazole's inhibition of diazepam metabolism. Clin Pharmacol Ther. 1995;58(1):62-72. 
12. Gugler R, Hartmann M, Rudi J, et al. Lack of pharmacokinetic interaction of pantoprazole with diazepam in man. Br J Clin Pharmacol. 1996;42(2):249-252.

13. Lefebvre RA, Flouvat B, Karolac-Tamisier S, Moerman E, Van Ganse E. Influence of lansoprazole treatment on diazepam plasma concentrations. Clin Pharmacol Ther. 1992;52(5):458-463.

14. Ishizaki T, Chiba K, Manabe K, et al. Comparison of the interaction potential of a new proton pump inhibitor, E3810, versus omeprazole with diazepam in extensive and poor metabolizers of S-mephenytoin 4'-hydroxylation. Clin Pharmacol Ther. 1995;58(2):155-164.

15. Naidu MUR, Shobha JC, Dixit VK, et al. Effect of multiple dose omeprazole on the pharmacokinetics of carbamazepine. Drug Invest. 1994;7(1):8-12.

16. Dixit RK, Chawla AB, Kumar N, Garg SK. Effect of omeprazole on the pharmacokinetics of sustained-release carbamazepine in healthy male volunteers. Methods Find Exp Clin Pharmacol. 2001;23(1):37-39.

17. Bertilsson L, Tybring G, Widén J, Chang M, Tomson T. Carbamazepine treatment induces the CYP3A4 catalysed sulphoxidation of omeprazole, but has no or less effect on hydroxylation via CYP2C19. Br J Clin Pharmacol. 1997;44(2):186-189.

18. Huber R, Bliesath H, Hartmann M, et al. Pantoprazole does not interact with the pharmacokinetics of carbamazepine. Int J Clin Pharmacol Ther. 1998;36(10):521-524

19. Dalton MJ, Powell JR, Messenheimer JA Jr. Ranitidine does not alter single-dose carbamazepine pharmacokinetics in healthy adults. Drug Intell Clin Pharm. 1985;19(12):941-944.

20. Han XM, Ouyang DS, Chen XP, et al. Inducibility of CYP1A2 by omeprazole in vivo related to the genetic polymorphism of CYP1A2. Br J Clin Pharmacol. 2002;54(5):540-543.

21. Rost KL, Brösicke H, Heinemeyer G, Roots I. Specific and dosedependent enzyme induction by omeprazole in human beings. Hepatology. 1994;20(5):1204-1212.

22. Frick A, Kopitz J, Bergemann N. Omeprazole reduces clozapine plasma concentrations. A case report. Pharmacopsychiatry. 2003; 36(3):121

23. Mookhoek EJ, Loonen AJ. Retrospective evaluation of the effect of omeprazole on clozapine metabolism. Pharm World Sci. 2004;26(3): 180-182.

24. Hartmann M, Zech K, Bliesath H, et al. Pantoprazole lacks induction of CYP1A2 activity in man. Int J Clin Pharmacol Ther. 1999;37(4): 159-164.

25. Bondolfi G, Morel F, Crettol S, Rachid F, Baumann P, Eap CB. Increased clozapine plasma concentrations and side effects induced by smoking cessation in 2 CYP1A2 genotyped patients. Ther Drug Monit 2005;27(4):539-543.

26. Falcon RW, Kakuda TN. Drug interactions between HIV protease inhibitors and acid-reducing agents. Clin Pharmacokinet. 2008;47(2):75-89.

27. Burger DM, Hugen PW, Kroon FP, et al. Pharmacokinetic interaction between the proton pump inhibitor omeprazole and the HIV protease inhibitor indinavir. AIDS. 1998;12(15):2080-2082.

28. Fang AF, Damle BD, LaBadie RR, Crownover PH, Hewlett D Jr, Glue PW. Significant decrease in nelfinavir systemic exposure after omeprazole coadministration in healthy subjects. Pharmacotherapy. 2008;28(1):42-50.

29. Zhu L, Persson A, Mahnke L, et al. Effect of low-dose omeprazole (20 mg daily) on the pharmacokinetics of multiple-dose atazanavir with ritonavir in healthy subjects. J Clin Pharmacol. 2011;51(3):368-377.

30. Sharma M, Saravolatz LD. Rilpivirine: a new non-nucleoside reverse transcriptase inhibitor. J Antimicrob Chemother. 2013;68(2):250-256

31. Tappouni HL, Rublein JC, Donovan BJ, et al. Effect of omeprazole on the plasma concentrations of indinavir when administered alone and in combination with ritonavir. Am J Health Syst Pharm. 2008;65(5):422-428.

32. Saah AJ, Winchell GA, Nessly ML, Seniuk MA, Rhodes RR, Deutsch PJ. Pharmacokinetic profile and tolerability of indinavir-ritonavir combinations in healthy volunteers. Antimicrob Agents Chemother. 2001;45(10):2710-2715.
33. Zhou S, Chan E, Li X, Huang M. Clinical outcomes and management of mechanism-based inhibition of cytochrome P4503A4. Ther Clin Risk Manag. 2005;1(1):3-13.

34. Boffito M, Carriero P, Trentini L, et al. Pharmacokinetics of saquinavir co-administered with cimetidine. J Antimicrob Chemother. 2002;50(6):1081-1084.

35. Saberi P, Ranatunga DK, Quesenberry CP, Silverberg MJ. Clinical implications of the nelfinavir-proton pump inhibitor drug interaction in patients with human immunodeficiency virus. Pharmacotherapy. 2011;31(3):253-261.

36. Luber AD, Brower R, Kim D, Silverman R, Peloquin CA, Frank I. Steady-state pharmacokinetics of once-daily fosamprenavir/ritonavir and atazanavir/ritonavir alone and in combination with $20 \mathrm{mg}$ omeprazole in healthy volunteers. HIV Med. 2007;8(7):457-464.

37. Van Heeswijk R, Hoetelmans R, Kestens D, et al; for: International AIDS Society; The pharmacokinetic (PK) interaction between famotidine and TMC278, a next generation non-nucleoside reverse transcriptase inhibitor (NNRTI), in HIV-negative volunteers. Proceedings of the Fourth IAS Conference on HIV Pathogenesis, Treatment, and Prevention; 2007; Sydney, Australia.

38. Brown KC, Paul S, Kashuba AD. Drug interactions with new and investigational antiretrovirals. Clin Pharmacokinet. 2009;48(4): 211-241.

39. Beorlegui B, Aldaz A, Ortega A, Aquerreta I, Sierrasesúmega L, Giráldez J. Potential interaction between methotrexate and omeprazole. Ann Pharmacother. 2000;34(9):1024-1027.

40. Bezabeh S, Mackey AC, Kluetz P, Jappar D, Korvick J. Accumulating evidence for a drug-drug interaction between methotrexate and proton pump inhibitors. Oncologist. 2012;17(4):550-554.

41. Bauters TG, Verlooy J, Robays H, Laureys G. Interaction between methotrexate and omeprazole in an adolescent with leukemia: a case report. Pharm World Sci. 2008;30(4):316-318.

42. Ranchon F, Vantard N, Gouraud A, et al. Suspicion of drug-drug interaction between high-dose methotrexate and proton pump inhibitors: a case report - should the practice be changed? Chemotherapy. 2011;57(3):225-229.

43. Christians U, Schmidt G, Bader A, et al. Identification of drugs inhibiting the in vitro metabolism of tacrolimus by human liver microsomes. Br J Clin Pharmacol. 1996;41(3):187-190.

44. Maguire M, Franz T, Hains DS. A clinically significant interaction between tacrolimus and multiple proton pump inhibitors in a kidney transplant recipient. Pediatr Transplant. 2012;16(6):E217-E220.

45. Hosohata K, Masuda S, Katsura T, et al. Impact of intestinal CYP2C19 genotypes on the interaction between tacrolimus and omeprazole, but not lansoprazole, in adult living-donor liver transplant patients. Drug Metab Dispos. 2009;37(4):821-826.

46. Hosohata K, Masuda S, Ogura Y, et al. Interaction between tacrolimus and lansoprazole, but not rabeprazole in living-donor liver transplant patients with defects of CYP2C19 and CYP3A5. Drug Metab Pharmacokinet. 2008;23(2):134-138.

47. Hosohata K, Masuda S, Yonezawa A, et al. Absence of influence of concomitant administration of rabeprazole on the pharmacokinetics of tacrolimus in adult living-donor liver transplant patients: a case-control study. Drug Metab Pharmacokinet. 2009;24(5):458-463.

48. Takahashi K, Yano I, Fukuhara Y, et al. Distinct effects of omeprazole and rabeprazole on the tacrolimus blood concentration in a kidney transplant recipient. Drug Metab Pharmacokinet. 2007;22(6):441-444.

49. Lorf T, Ramadori G, Ringe B, Schwörer H. The effect of pantoprazole on tacrolimus and cyclosporin A blood concentration in transplant recipients. Eur J Clin Pharmacol. 2000;56(5):439-440.

50. Doesch AO, Mueller S, Konstandin M, et al. Proton pump inhibitor co-medication reduces active drug exposure in heart transplant recipients receiving mycophenolate mofetil. Transplant Proc. 2010; 42(10):4243-4246.

51. David-Neto E, Takaki KM, Agena F, et al. Diminished mycophenolic acid exposure caused by omeprazole may be clinically relevant in the first week posttransplantation. Ther Drug Monit. 2012;34(3):331-336. 
52. Kiberd BA, Wrobel M, Dandavino R, Keown P, Gourishankar S. The role of proton pump inhibitors on early mycophenolic acid exposure in kidney transplantation: evidence from the CLEAR study. Ther Drug Monit. 2011;33(1):120-123.

53. Kees MG, Steinke T, Moritz S, et al. Omeprazole impairs the absorption of mycophenolate mofetil but not of enteric-coated mycophenolate sodium in healthy volunteers. J Clin Pharmacol. 2012;52(8):1265-1272.

54. Drepper MD, Spahr L, Frossard JL. Clopidogrel and proton pump inhibitors - where do we stand in 2012? World J Gastroenterol. 2012;18(18):2161-2171.

55. Norgard NB, Mathews KD, Wall GC. Drug-drug interaction between clopidogrel and the proton pump inhibitors. Ann Pharmacother. 2009;43(7):1266-1274.

56. Bhatt DL, Cryer BL, Contant CF, et al; for COGENT Investigators. Clopidogrel with or without omeprazole in coronary artery disease. N Engl J Med. 2010;363(20):1909-1917.

57. Bates ER, Lau WC, Angiolillo DJ. Clopidogrel-drug interactions. J Am Coll Cardiol. 2011;57(11):1251-1263.

58. Wu J, Jia LT, Shao LM, et al. Drug-drug interaction of rabeprazole and clopidogrel in healthy Chinese volunteers. Eur J Clin Pharmacol. 2013;69(2):179-187.

59. Neubauer H, Engelhardt A, Krüger JC, et al. Pantoprazole does not influence the antiplatelet effect of clopidogrel-a whole blood aggregometry study after coronary stenting. J Cardiovasc Pharmacol. 2010;56(1):91-97.

60. Frelinger AL 3rd, Lee RD, Mulford DJ, et al. A randomized, 2-period, crossover design study to assess the effects of dexlansoprazole, lansoprazole, esomeprazole, and omeprazole on the steady-state pharmacokinetics and pharmacodynamics of clopidogrel in healthy volunteers. J Am Coll Cardiol. 2012;59(14):1304-1311.

61. Tunggal P, Ng FH, Lam KF, Chan FK, Lau YK. Effect of esomeprazole versus famotidine on platelet inhibition by clopidogrel: a double-blind, randomized trial. Am Heart J. 2011;162(5):870-874.

62. Ng FH, Tunggal P, Chu WM, et al. Esomeprazole compared with famotidine in the prevention of upper gastrointestinal bleeding in patients with acute coronary syndrome or myocardial infarction. Am J Gastroenterol. 2012;107(3):389-396.

63. Gurbel PA, Bliden KP, Fort JG, et al. Spaced administration of PA 32540 and clopidogrel results in greater platelet inhibition than synchronous administration of enteric-coated aspirin and enteric-coated omeprazole and clopidogrel. Am Heart J. 2013;165(2):176-182.

64. Kiley CA, Cragin DJ, Roth BJ. Omeprazole-associated digoxin toxicity. South Med J. 2007;100(4):400-402.

65. Gabello M, Valenzano MC, Barr M, Zurbach P, Mullin JM. Omeprazole induces gastric permeability to digoxin. Dig Dis Sci. 2010; 55(5):1255-1263.

66. Collett A, Tanianis-Hughes J, Carlson GL, Harwood MD, Warhurst G. Comparison of P-glycoprotein-mediated drug-digoxin interactions in Caco-2 with human and rodent intestine: relevance to in vivo prediction. Eur J Pharm Sci. 2005;26(5):386-393.

67. Pauli-Magnus C, Rekersbrink S, Klotz U, Fromm MF. Interaction of omeprazole, lansoprazole and pantoprazole with P-glycoprotein. Naunyn Schmiedebergs Arch Pharmacol. 2001;364(6):551-557.

68. Hartmann M, Huber R, Bliesath $\mathrm{H}$, et al. Lack of interaction between pantoprazole and digoxin at therapeutic doses in man. Int JClin Pharmacol Ther. 1996;34(Suppl 1):S67-S71.

69. Jaruratanasirikul S, Sriwiriyajan S. Effect of omeprazole on the pharmacokinetics of itraconazole. Eur J Clin Pharmacol. 1998;54(2):159-161.

70. Johnson MD, Hamilton CD, Drew RH, Sanders LL, Pennick GJ, Perfect JR. A randomized comparative study to determine the effect of omeprazole on the peak serum concentration of itraconazole oral solution. $J$ Antimicrob Chemother. 2003;51(2):453-457.

71. Alffenaar JW, van Assen S, van der Werf TS, Kosterink JG, Uges DR. Omeprazole significantly reduces posaconazole serum trough level. Clin Infect Dis. 2009;48(6):839.
72. Lange D, Pavao JH, Wu J, Klausner M. Effect of a cola beverage on the bioavailability of itraconazole in the presence of $\mathrm{H} 2$ blockers. J Clin Pharmacol. 1997;37(6):535-540.

73. Dolton MJ, Ray JE, Marriott D, McLachlan AJ. Posaconazole exposure-response relationship: evaluating the utility of therapeutic drug monitoring. Antimicrob Agents Chemother. 2012;56(6):2806-2813.

74. Ross AL, Slain D, Cumpston A, Bryant AM, Hamadani M, Craig M. Evaluation of an alternative posaconazole prophylaxis regimen in haematological malignancy patients receiving concomitant stress ulcer prophylaxis. Int J Antimicrob Agents. 2012;40(6):557-561.

75. Ajmera AV, Shastri GS, Gajera MJ, Judge TA. Suboptimal response to ferrous sulfate in iron-deficient patients taking omeprazole. Am JTher. 2012;19(3):185-189.

76. Karam WG, Goldstein JA, Lasker JM, Ghanayem BI. Human CYP2C19 is a major omeprazole 5-hydroxylase, as demonstrated with recombinant cytochrome P450 enzymes. Drug Metab Dispos. 1996;24(10): 1081-1087.

77. Faucette SR, Zhang TC, Moore R, et al. Relative activation of human pregnane $\mathrm{X}$ receptor versus constitutive androstane receptor defines distinct classes of CYP2B6 and CYP3A4 inducers. J Pharmacol Exp Ther. 2007;320(1):72-80.

78. Chen Y, Ferguson SS, Negishi M, Goldstein JA. Identification of constitutive androstane receptor and glucocorticoid receptor binding sites in the CYP2C19 promoter. Mol Pharmacol. 2003;64(2):316-324.

79. Mugundu GM, Hariparsad N, Desai PB. Impact of ritonavir, atazanavir and their combination on the CYP3A4 induction potential of efavirenz in primary human hepatocytes. Drug Metab Lett. 2010;4(1):45-50.

80. Michaud V, Ogburn E, Thong N, et al. Induction of CYP2C19 and CYP3A activity following repeated administration of efavirenz in healthy volunteers. Clin Pharmacol Ther. 2012;91(3):475-482.

81. Wang LS, Zhou G, Zhu B, et al. St John's Wort induces both cytochrome P4503A4-catalyzed sulfoxidation and 2C19-dependent hydroxylation of omeprazole. Clin Pharmacol Ther. 2004;75(3):191-197.

82. Xie HG. Additional discussions regarding the altered metabolism and transport of omeprazole after long-term use of St John's Wort. Clin Pharmacol Ther. 2005;78(4):440-441.

83. Zuo XC, Zhang BK, Jia SJ, et al. Effects of Ginkgo biloba extracts on diazepam metabolism: a pharmacokinetic study in healthy Chinese male subjects. Eur J Clin Pharmacol. 2010;66(5):503-509.

84. Lei HP, Wang G, Wang LS, et al. Lack of effect of Ginkgo biloba on voriconazole pharmacokinetics in Chinese volunteers identified as CYP2C19 poor and extensive metabolizers. Ann Pharmacother. 2009;43(4):726-731.

85. Zadoyan G, Rokitta D, Klement S, et al. Effect of Ginkgo biloba special extract EGb761 ${ }^{\circledR}$ on human cytochrome P450 activity: a cocktail interaction study in healthy volunteers. Eur J Clin Pharmacol. 2012; 68(5):553-560.

86. Yin OQ, Tomlinson B, Waye MM, Chow AH, Chow MS. Pharmacogenetics and herb-drug interactions: experience with Ginkgo biloba and omeprazole. Pharmacogenetics. 2004;14(12): 841-850.

87. Huang W, Zhang J, Moore DD. A traditional herbal medicine enhances bilirubin clearance by activating the nuclear receptor CAR. J Clin Invest. 2004;113(1):137-143.

88. Fan L, Wang G, Wang LS, et al. Herbal medicine yin zhi huang induces CYP3A4-mediated sulfoxidation and CYP2C19-dependent hydroxylation of omeprazole. Acta Pharmacol Sin. 2007;28(10): 1685-1692.

89. Pace F, Pallotta S, Casalini S, Porro GB. A review of rabeprazole in the treatment of acid-related diseases. Ther Clin Risk Manag. 2007; 3(3):363-379.

90. Marelli S, Pace F. Rabeprazole for the treatment of acid-related disorders. Expert Rev Gastroenterol Hepatol. 2012;6(4):423-435.

91. Moher D, Liberati A, Tetzlaff J, Altman DG; for PRISMA Group. Preferred reporting items for systematic reviews and meta-analyses: the PRISMA statement. BMJ. 2009;339:b2535. 
Therapeutics and Clinical Risk Management

Dovepress

\section{Publish your work in this journal}

Therapeutics and Clinical Risk Management is an international, peerreviewed journal of clinical therapeutics and risk management, focusing on concise rapid reporting of clinical studies in all therapeutic areas outcomes, safety, and programs for the effective, safe, and sustained use of medicines. This journal is indexed on PubMed Central, CAS
EMBase, Scopus and the Elsevier Bibliographic databases. The manuscript management system is completely online and includes a very quick and fair peer-review system, which is all easy to use. Visit http://www.dovepress.com/testimonials.php to read real quotes from published authors.

Submit your manuscript here: http://www.dovepress.com/therapeutics-and-clinical-risk-management-journal 